\title{
The effect of a wheelchair designed to prevent forward head posture on swallowing duration and integrated electromyography of suprahyoid muscles
}

\author{
Hiroshi Goda ${ }^{*}$, Tatsuo Hatta ${ }^{1}$, Kishigami Hirotoshi ${ }^{1}$, Tamotsu Ikeda ${ }^{2}$, Satomi Yamada ${ }^{2}$ and Yoshihiko Shibukawa ${ }^{2}$ \\ ${ }^{1}$ Japan Health Care College, Eniwa, Japan \\ ${ }^{2}$ Nihon Welfare Rehabilitation School, Eniwa, Japan
}

\begin{abstract}
Aspiration is a severe problem for elderly patients with disabilities. The causes of aspiration include forward head posture and muscle weakness. Forward Head Posture leads to a chin-up posture and affects the activity of the suprahyoid muscles. This study aimed to assess the utility of a wheelchair incorporating an increased seat to back support angle to improve swallowing. The study cohort included 21 healthy individuals $(9$ males and 12 females; mean age $=20.8 \pm 0.9$ years). Two wheelchairs were used in this study: model RX-1 and model RX_ABS Lo, which is a modification of RX-1 with a different back support system. Subjects were instructed to sit in each wheelchair and swallow $15 \mathrm{~mL}$ of water. Suprahyoid muscle activity was measured in a resting position and during swallowing, while posture in the sagittal plane was recorded using a digital video camera. We also measured buttock and back support pressure distribution. Swallowing duration, integrated electromyography, posture and pressure distribution between the two wheelchairs were compared using paired-samples $t$-tests. After the exclusion of five subjects, 16 were included for analysis. Swallowing duration and integrated electromyography significantly were decreased with RX_ABS Lo compared to RX-1. Head and neck alignment were more erect and trunk alignment more inclined in RX_ABS Lo compared to RX-1. RX_ABS Lo led to decreased buttock pressure and increased back support pressure; further, the improved forward head posture observed with RX_ABS Lo facilitated better chin alignment. In conclusion, use of RX_ABS Lo resulted in decreased swallowing duration and integrated electromyography due to improvement in forward head posture.
\end{abstract}

\section{Introduction}

The population of Japan is aging at a rate of $27 \%$ [1]. The growing elderly population has led to an increase in the number of residents living in long-term care facilities [2]. The act of eating is important to retain independence for elderly individuals with disabilities [3], and swallowing disorders thus represent a severe problem among disabled elders living in long-term care facilities [4]. Aspiration may cause pneumonia and can be fatal among elderly individuals.

The causes of aspiration include weakening of the suprahyoid muscles due to age, dysphagia as a result of stroke, and forward head posture (FHP). FHP describes head and neck alignment forward of the trunk. Therefore, to swallow water, individuals close the mouse with their chin-up posture. In the swallowing phase, by contracting the suprahyoid muscle, the larynx is protected and the pharynx opens. Chin-up posture extends the suprahyoid muscle as well as opening the larynx. Therefore, even in healthy individuals, swallowing while in the chin-up posture increases the movement distance and decreases the maximum acceleration of the hyoid bone [5]. As a result, in healthy individuals, swallowing duration is prolonged and integrated electromyography (iEMG) of suprahyoid muscles is increased [6]. In elderly or disabled individuals, muscle weakness and paralysis of the suprahyoid muscles may be observed. As such, swallowing duration and iEMG may be further increased.

As a treatment for dysphagia, rehabilitation professionals may provide exercises to strengthen the suprahyoid muscles and/or coach patients to improve swallowing posture [7-9]. On the other hand, occupational therapists recommended chin-down posture is recommended for swallowing [10]. In this posture, swallowing duration is shortened and iEMG is decreased [6]. However, in hospitals and long-term care facilities, most elderly disabled individuals do not engage in the recommended posture, instead swallowing while in the chin-up posture. This is largely due to the back support structure of the standard wheelchair, which leads to a chin-up posture. Unfortunately, most elderly individuals use a standard wheelchair, in which the back support is attached vertically to the buttock. This linear back support touches a small area of the kyphosis, or curvature of the spine. The sling seat that stretches between the back support structure has low shrinkage, causing the individual's trunk to be pushed forward. This results in FHP and chin-up posture, even among healthy individuals.

We developed a wheelchair that incorporates pelvic support. The structure of back support is different from standard type wheelchairs. The posts of back support are inclined at two points. Additionally, at each region of pelvic and thoracic, support belt is stretched like an arc when viewed from above [11]. We hypothesized that as pelvic support belt prevented the posterior tilt of the pelvis, and the thoracic support belt prevented pushing the trunk forward, so that FHP was improved.

Correspondence to: Hiroshi Goda, Megumino Nishi-6 chome, 061-1373 Eniwa, Japan, Tel: +081 123-29-3171; Fax: +081 123-29-3171

Key words: wheelchair, forward head posture, swallowing, EMG, suprahyoid

Received: June 02, 2017; Accepted: June 23, 2017; Published: June 26, 2017 

muscles

We previously confirmed the effect of back support that FHP for healthy and elderly individuals is improved using the wheelchair incorporating the pelvic support belt [12-14]. Furthermore, in our previous research, a wheelchair incorporating a pelvic support belt prevented FHP in elderly disabled individuals for a 30 minute duration [14]. We next wished to examine whether preventing FHP using the wheelchair incorporating a pelvic support belt influenced position of chin. In addition, we believe this wheelchair influenced swallowing duration and/or iEMG. However, as mentioned above, aspiration among elderly persons with disabilities is affected by muscle weakness and paralysis of the suprahyoid muscles. Therefore, we divided this project into three stages; first stage is preventing FHP, second stage is preventing FHP for elderly individuals, and third stage is preventing FHP for elderly disabled individuals. For this reason, as an initial stage of the project, we decided to examine whether swallowing duration and iEMG were reduced via the prevention of FHP among healthy individuals without suprahyoid muscle weakness or paralysis. The purpose of the current study was therefore to test whether preventing FHP using a wheelchair incorporating a pelvic support belt reduces swallowing duration and iEMG among healthy subjects.

\section{Materials and methods}

\section{Participants}

This study was a prospective, randomized, cross-over trial design. Although individuals sit on two wheelchairs, the order is random. Inclusion criterion was that the adult ( $>20$ years) had no disturbance of oral function, and exclusion criterion was that adult could not sit on the wheelchair with their thigh parallel to floor. Additionally, we posted an advertisement catalogue on the bulletin board of the university where researchers work. The study cohort included 21 healthy individuals ( 9 males and 12 females; mean age $=20.8 \pm 0.9$ years) who met the inclusion criteria. Experiment was performed in our experiment room on August $4^{\text {th }}-6^{\text {th }}$ and October $26^{\text {th }}-29^{\text {th }} 2015$.

The mean height and weight of participants were $159 \pm 0.2 \mathrm{~cm}$ and $56.8 \pm 9.5 \mathrm{~kg}$, respectively. Ethical approval was obtained from the Ethics Review Committee, Faculty of Health Sciences, Hokkaido University [15-18] and conformed to the Declaration of Helsinki (1996). Written informed consent was obtained from each participant prior to testing (Figure 1).

\section{Wheelchairs (models RX_ABS Lo and RX-1)}

Two wheelchairs were used in this study (Figure 2 and 3) [14]. Model RX_ABS Lo is a modification of model RX-1 with a different back support system (both wheelchairs were produced by $\mathrm{MiKi}$ Kogyosho Co., Ltd., Nagoya, Japan). In RX_ABS Lo, the angles between the back supports and sitting surfaces are $106^{\circ}$ and $130^{\circ}$, respectively. The pelvic support belt is at a height of $18 \mathrm{~cm}$ and forms an arc when viewed from above. The pelvic support belt of RX_ABS Lo supports the iliac crest from the sides, and the pelvis and lower thoracic region from the back. At the level of the push handles, two thoracic support belts are stretched across to form a ladder. In the standard setting, the thoracic support belt is stretched up to 1 inch (approximately $2.54 \mathrm{~cm}$ ) behind the pelvic support belt. Although the RX_ABS Lo can be adjusted to accommodate individuals with varying body sizes, the standard setting was used in this study.

RX-1 was used as a control because it has a structural design identical to RX_ABS Lo, with the exception of the back-support structure. The angles of the back support and armrests with respect to the sitting surface are $98^{\circ}$ and $115^{\circ}$, respectively. Five 5-cm back support

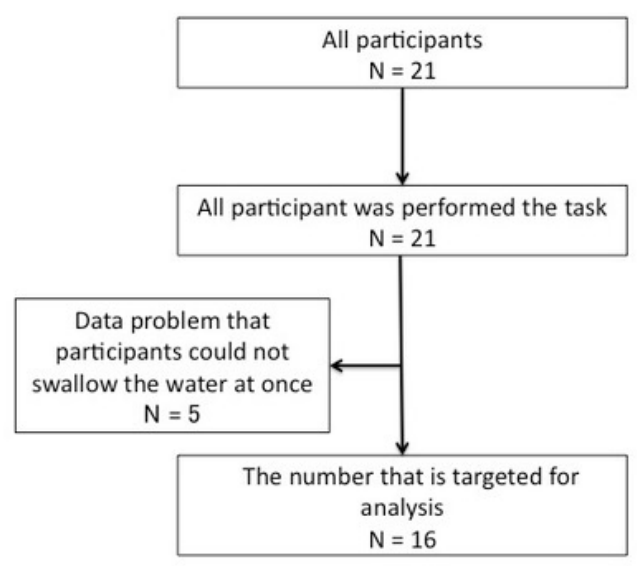

Figure1. Flow of this experiment (a)

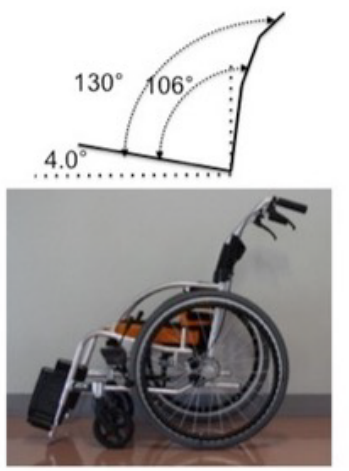

(b)

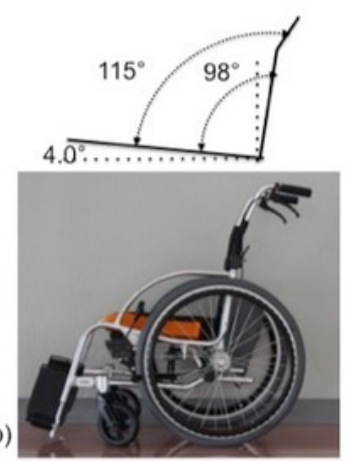

Figure 2. The novel- developed product wheel chair (RX ABS Lo) and control wheel chair (RX_1) (RX_ABS Lo) has pelvic support belt and thoracic support belt (a). RX-1, (b) has five back support belt like ladder.

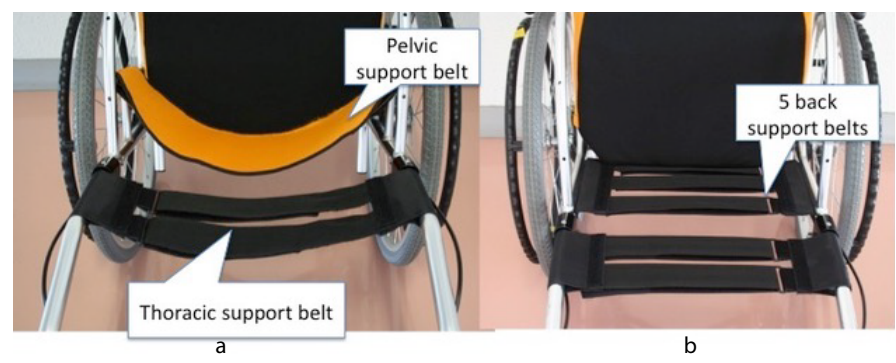

Figure 3. Two type of wheel chair we used in this experiment (Horizontal Plane) The area and height between RX_ABS Lo (a) and RX-1(b) are same. RX_ABS Lo has pelvic support belt and thoracic support belt (a). RX-1 has thoracic support belt. The belt is stretched tight.

belts are stretched between the posts of the back support, resembling a ladder. Most disabled elderly individuals use standard wheelchairs consisting of a sling seat without an adjustable belt. Therefore, in this study, the five support belts were set at high tension to mimic standard wheelchairs. The same cushions and back covers were used for both the RX_ABS Lo and RX-1 models.

\section{Initial seating positions and instructions}

Individuals were seated with the pelvis in contact with wheelchair back supports. Pelvic position was confirmed by manual palpation. Additionally, individuals were instructed to use the foot supports. We assumed all individuals used both back supports, but participants 

muscles

had enough mobility and muscle power. Therefore, to display usual posture, individuals were made to sit so that the following occurred:

1) Flexion of the trunk so that the chest makes contact with the thigh.

2) The trunk is extended to ensure comfort.

3) The upper region of back support can be used if needed.

Study participants were instructed to swallow $15 \mathrm{~mL}$ of water at once without performing a chewing motion $[15,16]$.

\section{Electromyography}

Electromyography (EMG) was used to record the suprahyoid muscle group, which is used to elevate the pharynx during swallowing [17-20]. Activity of the suprahyoid muscle group was measured using surface electrodes (Biometrics Ltd., Newport, UK) set apart at a distance of $20 \mathrm{~mm}$. Two surface electrodes were attached to the skin beneath the chin on both side of the midline to record submental myoelectrical activity [17]. The sampling rate was $1000 \mathrm{~Hz}$. Signals from the EMG electrodes were amplified and filtered (low: $5 \mathrm{~Hz}$; high: $500 \mathrm{kHz}$ ) to remove movement-related artifacts.

The thresholds for the onset and offset of swallow-related EMG bursts were defined as follows: for a mean value at rest, $5 \mathrm{~s}$ was adopted as a control. When trial values exceeded the control by $>2$ standard deviations, the EMG burst was considered active. The start and end of the swallowing event were defined as the appearance and disappearance of the EMG burst, respectively [6, 21, 22]. iEMG was calculated to obtain muscle activity.

\section{Determination of posture}

Wheelchair sitting postures were recorded using a megapixel digital camera (Canon iVIS HF M43; Cannon Corporation, Tokyo, Japan), which was fitted on a tripod located $3 \mathrm{~m}$ from the wheelchair [23]. To enable posture determination, surface landmarks of the neck and trunk were identified and marked by placing adhesive reflective balls on the $\mathrm{C} 7$ spinous process, tragus of one ear, and great trochanter using double-sided tape. The following postural angles were measured: head, cervical, trunk, head joint, and cervical joint (Table 1). The position of each marker was recorded and digitized using DARTFISH software (Dartfish Co., Ltd., Lausanne, Switzerland) [14, 24].

\section{Measurement of back support and buttock pressures}

The SR soft vision pressure-mapping system (Sumitomo Riko Company Limited, Tokyo, Japan) was used to assess the pressure of both back support and buttocks [14]. Dedicated software was used to calculate the total pressure and distribution of the sensing area of each mat. A representative value was calculated as the average of $30 \mathrm{~s}$ for each individual.

Table 1. Operational definitions for posture analysis

\begin{tabular}{|l|l|}
\hline \multicolumn{1}{|c|}{ Dimension of posture (degree) } & \multicolumn{1}{c|}{ Operational definition } \\
\hline Head angle & $\begin{array}{l}\text { The angle between the line from the lateral orbital } \\
\text { rim to the tragus and horizontal }\end{array}$ \\
\hline Cervical angle & $\begin{array}{l}\text { The angle between the line from the tragus to the } \\
\text { C7 spinous process and horizontal }\end{array}$ \\
\hline Trunk angle & $\begin{array}{l}\text { The angle between the C7 spinous and the great } \\
\text { trochanter and horizontal }\end{array}$ \\
\hline Head joint angle & $\begin{array}{l}\text { The angle between the line lateral orbital rim } \\
\text { - tragus and the line connecting the tragus and } \\
\text { orbital rim - tragus - C7 spinous process }\end{array}$ \\
\hline Cervical joint angle & $\begin{array}{l}\text { The angle between the line tragus - C7 spinous } \\
\text { process and the line connecting the C7 spinous } \\
\text { process - great trochanter }\end{array}$ \\
\hline
\end{tabular}

\section{Statistical analysis}

Comparisons between values of RX_ABS Lo and RX-1 were performed using paired-samples $t$-tests with a significance level of $p$ $<0.05$. All statistical analyses were performed using SPSS software version 22.0 (IBM SPSS, Inc., Chicago, IL, USA).

\section{Results}

\section{Participant characteristics}

Five participants could not swallow water at once; therefore, their data were excluded. A total of 16 participants participated in this study (mean age $=20.8 \pm 0.9$ years; mean height $=159.6 \pm 9.2 \mathrm{~cm}$; and mean body weight $=57.5 \pm 9.5 \mathrm{~kg}$ ). Power is 0.77 that is calculated using effect size $=0.5, \beta / \alpha$ ratio $=1$, and total sample size $=16$.

\section{Comparison of swallowing duration and iEMG in RX_ABS Lo vs. RX-1}

Typical data of EMG for each wheelchair are shown in Figure 4. Swallowing duration was significantly shorter with RX_ABS Lo than with RX-1 $(p<0.01)$. In addition, iEMG was significantly lower with RX_ABS Lo than with RX-1 $(p=0.02)$ (Table 2).

\section{Comparison of posture with RX_ABS Lo vs. RX-1}

Posture, cervical alignment, and mandibular alignment data are shown in Figure 5 Table 3. Trunk and cervical angles were significantly larger with RX_ABS Lo than with RX-1 $(p<0.01)$. Head joint and cervical angles were significantly smaller for RX_ABS Lo than for RX-1 $(p<0.05$ for both).

\section{Comparison of pressure distribution with $\mathrm{RX} \_\mathrm{ABS}$ Lo $v s$. RX-1}

Comparative results of buttock pressure and back support pressure in RX_ABS Lo versus RX-1 are shown in Table 4. Total back support pressure and sensor area were significantly larger for RX_ABS Lo than for RX-1 ( $p<0.01$, respectively).

\section{Discussion}

The purpose of this study was to determine whether use of a wheelchair incorporating a pelvic support belt decreased swallowing duration and iEMG via prevention of FHP. The structure of back support of RX_ABS Lo is absolutely different from standard type wheelchair. The back supports are inclined at two points. In addition, the support belt is stretched like an arc.

Results indicated that swallowing duration and iEMG were significantly reduced with RX_ABS Lo compared to RX-1. The following section discusses the effect and mechanism of reduced swallowing duration and iEMG observed with the RX_ABS Lo model.

\section{Effect of RX_ABS Lo on swallowing}

Swallowing duration and iEMG were reduced with use of the RX ABS Lo wheelchair. Participants consisted of healthy volunteers who sat on the two wheelchairs on separate days, therefore keeping fatigue of the suprahyoid muscles to a minimum. In other words, the changes in swallowing duration and iEMG were affected by postural changes.

A chin-up posture leads to prolonged swallowing duration and increases iEMG [6]. In the standard wheelchair model (RX-1), the cervical angle was smaller and the head joint angle larger than in the newly-developed model (RX_ABS Lo). This posture showed FHP and a 
Goda H (2017) The effect of a wheelchair designed to prevent forward head posture on swallowing duration and integrated electromyography of suprahyoid muscles

$\mathrm{RX}-1$

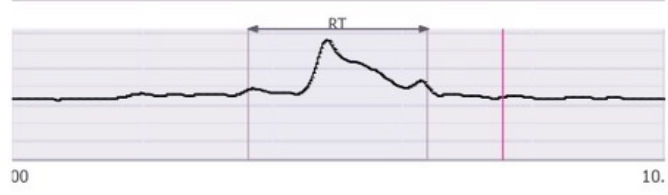

RX_ABS Lo

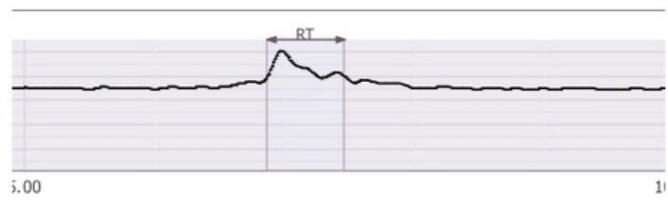

Figure 4. Muscle activity of suprahyoid muscle in typical case. A double - headed arrow indicate the swallowing duration. Swallowing duration in RX_ABS Lo is shorter than $\mathrm{RX}-1$. Also muscle activity in RX_ABS Lo is smaller than in RX-1

(a)

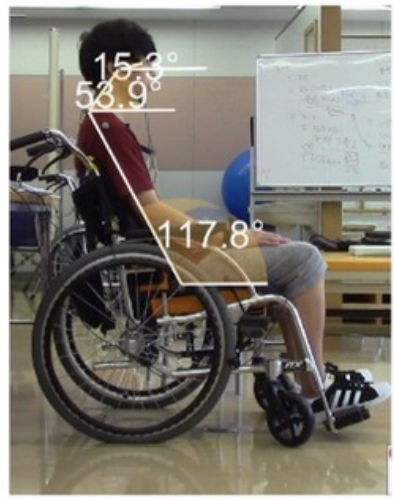

(b)

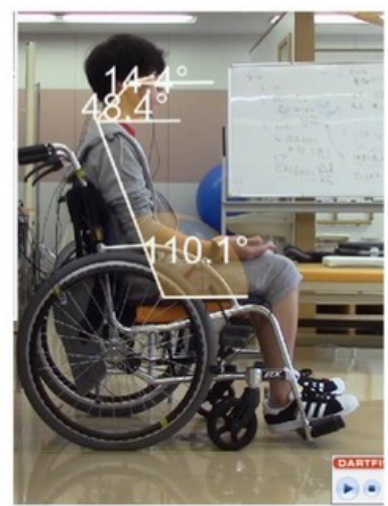

Figure 5. Swallowing posture in the novel-developed product wheel chair (RX_ABS Lo) and control wheelchair (RX-1). The trunk in RX_ABS Lo (a) is incline compared with RX1(b). And head and neck alignment in RX_ABS Lo is erect compared with RX-1

Table 2. Swallowing duration and integrated electromyography in RX_ABS Lo vs. RX-1 wheelchair models

\begin{tabular}{|l|l|l|l|l|}
\hline $\begin{array}{l}\text { EMG/pressure } \\
\text { distribution }\end{array}$ & $\begin{array}{l}\text { Right or left } \\
\text { suprahyoid } \\
\text { muscle }\end{array}$ & RX_ABS Lo & RX-1 & $\boldsymbol{p}$-value \\
\hline Swallowing duration (s) & Right & $0.97 \pm 0.42$ & $1.62 \pm 0.94$ & 0.00 \\
\hline & Left & $1.02 \pm 0.16$ & $1.64 \pm 0.22$ & 0.04 \\
\hline iEMG (mVs) & Right & $0.02 \pm 0.003$ & $0.03 \pm 0.005$ & 0.02 \\
\hline & Left & $0.03 \pm 0.006$ & $0.04 \pm 0.011$ & 0.02 \\
\hline
\end{tabular}

$\mathrm{iEMG}=$ integrated electromyography

Table 3. Sitting posture in RX_ABS Lo vs. RX-1 wheelchair models

\begin{tabular}{|l|l|l|l|}
\hline Dimension of posture (degree) & RX_ABS Lo & RX-1 & $p$-value \\
\hline Head angle & $20.3 \pm 1.5$ & $20.4 \pm 1.7$ & 0.96 \\
\hline Cervical angle & $47.4 \pm 1.3$ & $44.1 \pm 1.3$ & 0.00 \\
\hline Trunk angle & $119.3 \pm 1.5$ & $110.6 \pm 0.9$ & 0.00 \\
\hline Head joint angle & $151.9 \pm 2.0$ & $154.7 \pm 2.0$ & 0.03 \\
\hline Cervical joint angle & $108.7 \pm 1.8$ & $116.1 \pm 5.4$ & 0.00 \\
\hline
\end{tabular}

chin-up posture $[5,25]$. Additionally, it increased swallowing duration and iEMG.

In contrast, the cervical angle was improved and erect head and neck alignment were achieved using the RX_ABS Lo model. These findings indicate that RX_ABS Lo prevents FHP. Moreover, a reduced head joint angle facilitates chin-down posture. This posture displayed recommended posture in rehabilitation to prevent aspiration. In the chin-down posture, the opening of the larynx is narrow and that of the pharynx is wide, facilitating swallowing [25]. In addition, as the chin-
Table 4. Pressure distribution in RX_ABS Lo vs. RX-1 wheelchair models

\begin{tabular}{|l|l|l|l|l|}
\hline $\begin{array}{l}\text { Pressure } \\
\text { distribution } \\
(\mathbf{m m H g})\end{array}$ & $\begin{array}{l}\text { Total } \\
\text { pressure } \\
(\mathbf{m m H g})\end{array}$ & RX_ABS Lo & RX-1 & $\boldsymbol{p}$-value \\
\hline $\begin{array}{l}\text {-sensor } \text { area } \\
\left(\mathbf{c m}^{2}\right)\end{array}$ & $\begin{array}{l}\text { Total } \\
\text { pressure }\end{array}$ & $7057.34 \pm 326.94$ & $7785.78 \pm 331.77$ & 0.00 \\
\hline Buttock pressure & Sensor area & $786.61 \pm 33.15$ & $876.86 \pm 35.28$ & 0.00 \\
\hline Back support pressure & $\begin{array}{l}\text { Total } \\
\text { pressure }\end{array}$ & $1737.59 \pm 208.40$ & $889.88 \pm 147.23$ & 0.00 \\
\hline & Sensor area & $306.30 \pm 29.27$ & $159.19 \pm 23.72$ & 0.00 \\
\hline & & & & \\
\hline
\end{tabular}

down posture ensures shorting length of the suprahyoid muscle, ease to perform muscle strength. [26]. This minimizes the vertical distance and increases the maximum acceleration of the hyoid bone [5], reducing both swallowing duration and iEMG.

\section{Mechanism of preventing FHP on back support in RX_ABS Lo}

The cervical angle of RX_ABS Lo is larger than that of RX-1. The only difference between the two wheelchairs is the back-support design; namely, the angle of the back support of RX_ABS Lo is more inclined compared to that of RX-1. Horton et al. reported that inclined back support benefited head and neck alignment [27]. Therefore, tilting the back support of RX_ABS Lo promoted erect alignment of the head and neck. However, simply tilting the back support leads to posterior tilt of the pelvis [28]. Finally, posterior tilt of the pelvis exaggerates kyphosis [29], leading to FHP. Therefore, both a pelvic support belt and a thoracic support belt preventing posterior tilt of the pelvis and FHP are necessary ergonomic components of a wheelchair.

In our previous study, wheelchair incorporating pelvic support belt prevents FHP for elderly disabled individuals [14]. Their trunk is more inclined and head and neck alignment is more erect. Moreover, in the pressure distribution of wheelchair incorporating pelvic support belt, total back support pressure is increased and total buttock pressure is decreased. In addition, in the back support, total sensor area is increased. Moreover, our result supported previous research.

\section{Limitations}

We analyzed the static posture on swallowing. Because we could not discuss about the movement of swallowing, we regard this point as a limitation. Additionally, outcomes were tested in healthy adults only and results therefore cannot be generalized to other populations.

\section{Conclusions}

A novel wheelchair model that the back support is inclined and stretched, pelvic support belt reduced swallowing duration and iEMG via prevention of FHP. This wheelchair has the potential to prevent aspiration. Our next step will be to determine the effect of this wheelchair on swallowing duration and iEMG among elderly, disabled individuals who may experience suprahyoid muscle weakness or paralysis.

\section{Conflict of interest statement}

None of the authors of the current manuscript (Gouda, Hatta, Kishigami, Ikeda, Yamada, and Shibukawa) have any financial or personal relationships with other individuals or organizations that can inappropriately influence (bias) this work. 
Goda H (2017) The effect of a wheelchair designed to prevent forward head posture on swallowing duration and integrated electromyography of suprahyoid muscles

\section{References}

1. Statistical Handbook of Japan (2016)

2. Miura H, Yamasaki K, Kariyasu M, Miura K, Sumi Y (2003) Relationship between cognitive function and mastication in elderly females. J Oral Rehabil 30: 808-811.

3. Gerrard P (2013) The hierarchy of the activities of daily living in the Katz index in residents of skilled nursing facilities. J Geriatr Phys Ther 36: 87-91. [Crossref]

4. Manabe T, Teramoto S, Tamiya N, Okochi J, Hizawa N (2015) Risk Factors for Aspiration Pneumonia in Older Adults. PLoS One 10: e0140060. [Crossref]

5. Leigh JH, Oh BM, Seo HG, Lee GJ, Min Y, et al. (2015) Influence of the chin-down and chin-tuck maneuver on the swallowing kinematics of healthy adults. Dysphagia 30: 89-98. [Crossref]

6. Sakuma T and Kida I (2010) Relationship between ease of swallowing and deglutitionrelated muscle activity in various postures. J Oral Rehabil 37: 583-589.

7. Park JS, Oh DH, Chang MY (2016) Effect of expiratory muscle strength training on swallowing-related muscle strength in community-dwelling elderly individuals: a randomized controlled trial. Gerodontol 34: 124-128.

8. Athukorala RP, Jones RD, Sella O, Huckabee ML (2014) Skill training for swallowing rehabilitation in patients with Parkinson's disease. Arch Phys Med Rehabil 95: 1374-1382.

9. Wada S, Tohara H, Iida T, Inoue M, Sato M, et al (2012) Jaw-opening exercise for insufficient opening of upper esophageal sphincter. Arch Phys Med Rehabil 93: 1995-1999.

10. Woo HS, Park SH, Jung MY, Yoo EY, Park JH (2012) The effects of cranio-cervical flexion on activation of swallowing-related muscles. J Oral Rehabil 39: 805-811. [Crossref]

11. Nishimura S (2013) Science of the wheelchair and humans. Description of pelvic support model in active balance seating. Hokkaido J Occup Ther 30: 8-20.

12. Sawada N, Hatta T, Kishigami H, Shimizu M, Yoda T, et al (2015).The effect of a newly developed wheelchair with thoracic and pelvic support on cervical movement and muscle activity in healthy elderly women. Eur Geriatr Med 6: 286-290.

13. Hatta T, Kishigami H, Goda H, Sawada N (2015) Does ABS wheelchair improve the sitting posture of clients with Hemiplegia? A pilot study. Rehabil Eng 30: 28-31.

14. Goda H, Hatta T, Kishigami H, Suzuki A, Ikeda T (2015) Does a Novel-Developed Product of Wheelchair Incorporating Pelvic Support Prevent Forward Head Posture during Prolonged Sitting? PLoS One 10: e142617. [Crossref]

15. Hori K, Tamine K, Barbezat C, Maeda Y, Yamori M, et al (2011) Influence of chindown posture on tongue pressure during dry swallow and bolus swallows in healthy subjects Dysphagia 26: 238-245.
16. Belo LR, Gomes NA, Coriolano M, de Souza ES, Moura DA, et al (2014) The relationship between limit of Dysphagia and average volume per swallow in patients with Parkinson's disease Dysphagia 29: 419-424.

17. Hiramatsu T, Kataoka H, Osaki M, Hagino H (2015) Effect of aging on oral and swallowing function after meal consumption. Clin Interv Aging 10: 229-235. [Crossref]

18. Kim HR, Lee SA, Kim K, Leigh JH, Han TR, et al (2015) Submental muscle activity is delayed and shortened during swallowing following stroke. PM R 7: 938-945.

19. Watts CR (2013) Measurement of hyolaryngeal muscle activation using surface electromyography for comparison of two rehabilitative dysphagia exercises. Arch Phys Med Rehabil 94: 2542-2548.

20. Ertekin C, Keskin A, Kiylioglu N, Kirazli Y, On AY, et al. (2001) The effect of head and neck positions on oropharyngeal swallowing: a clinical and electrophysiologic study. Arch Phys Med Rehabil 82: 1255-1260. [Crossref]

21. Yamamoto S, Taniguchi H, Hayashi H, Hori K, Tsujimura T, et al (2014) How do tablet properties influence swallowing behaviors?. J Pharm Pharmacol 66: 32-39.

22. Inagaki D, Miyaoka Y, Ashida I, Ueda K, Yamada Y (2007) Influences of body posture on duration of oral swallowing in normal young adults. J Oral Rehabil 34: 414-421. [Crossref]

23. Yoo W-G, An D-H (2009) The relationship between the active cervical range of motion and changes in head and neck posture after continuous VDT work. Industrial Health 47: 183-188.

24. Khadilkar L, MacDermid JC, Sinden KE, Jenkyn TR, Birmingham TB, et al (2014) An analysis of functional shoulder movements during task performance using Dartfish movement analysis software. Int J Shoulder Sur 8: 1-9.

25. Welch MV, Logemann JA, Rademaker AW, Kahrilas PJ (1993) Changes in pharyngeal dimensions effected by chin tuck. Arch Phys Med Rehabil 74: 178-181. [Crossref]

26. Greene DP, Roberts SL (2004) Kinesiology: Movement in the Context of Activity. Mosby.

27. Horton SJ, Johnson GM, Skinner MA (2010) Changes in head and neck posture using an office chair with and without lumbar roll support. Spine 35: E542-548.

28. Park UJ, Jang SH (2011) The influence of backrest inclination on buttock pressure. Ann Rehabil Med 35: 897-906. [Crossref]

29. Gardocki RJ, Watkins RG, Williams LA (2002) Measurements of lumbopelvic lordosis using the pelvic radius technique as it correlates with sagittal spinal balance and sacral translation. Spine 2: 421-429.

Copyright: (C2017 Goda H. This is an open-access article distributed under the terms of the Creative Commons Attribution License, which permits unrestricted use, distribution, and reproduction in any medium, provided the original author and source are credited. 